\title{
Film cooling with swirling coolant flow
}

\author{
K. Takeishi, Y. Oda, Y. Egawa \& T. Kitamura \\ Department of Mechanical Engineering, Osaka University, Japan
}

\begin{abstract}
This paper describes the experimental results of a new film cooling method blowing through circular and shaped film cooling holes with swirling coolant flow. The experiments have been conducted by using a low-speed wind tunnel. The film cooling effectiveness on the flat wall was measured by using pressure sensitive paint (PSP) techniques. In addition, the spatial distribution of the film cooling effectiveness and flow field were measured by leaser induced fluorescence (LIF) and particle image velocimetry (PIV), respectively. In the case of the circular film cooling hole, the coolant jet penetration into the mainstream is suppressed by the swirling motion of the coolant. As a result, although the coolant jet is deflected in the pitch direction, the film cooling effectiveness distribution on the wall keeps a higher value behind the cooling hole over a long range. Additionally, the anti-kidney vortex structure disappeared. For the shaped cooling hole, the coolant jet spreads wider in the spanwise direction at the downstream. Thus, the pitch averaged film cooling effectiveness at the downstream was $50 \%$ higher than that of the non-swirling case.
\end{abstract}

Keywords: gas turbine, heat transfer, film cooling, PSP, LIF, PIV.

\section{Introduction}

Gas turbines are used for aircraft propulsion and land-based power generation. Developments in turbine cooling technology play a critical role in increasing the thermal efficiency and power output of advanced gas turbines. Gas turbine blades are cooled externally by film cooling. This is achieved by injecting relatively cooler air from the internal coolant passages out of the blade surface in order to form a protective layer between the blade surface and hot gas-path flow. The interaction between the film-cooling air and the mainstream, which is 
representative of film cooling, forms a shear layer that leads to mixing and a decay of the cooling performance along a blade surface.

It is important to improve the film cooling effectiveness by minimizing the film cooling air flow rate. The cooling air blowing through an inclined circular hole penetrates into the mainstream and generates an anti-kidney vortex structure by the interaction between the mainstream and the film cooling jet. The downwash of hot mainstream gas and catching the hot gas below the film cooling air jet decreases drastically the film cooling effectiveness on turbine blade surfaces. Shaped film cooling is one of the most attractive inventions by the incorporation of exit shaping to the film holes, which results in lower momentum coolant injection jets with greater surface coverage [9].

There have been many studies on film cooling. A review paper by Goldstein [1] summarized early works in this area and Bogard and Thole [2] reviewed the latest one. Early investigations on film cooling were flows blowing through inclined discrete holes, including Eriksen and Goldstein [3] and Bernsdorf et al. [4]. Efforts to improve film cooling efficiency by adopting film cooling holes with expanded exits have been made experimentally by Goldstein and Eckert [5], Bell et al. [6], Takeishi and Aoki. [7] and Yu et al. [8]. An excellent summary paper on shaped film cooling was published by Bunker [9].

Several new ideas to control the anti-kidney vortex structure are presented. Kusterer et al. [10] investigated two film cooling arrangements with different compound angles, and Heidmann and Ekkad [11] proposed additional cooling air jets in a circular film cooling hole to control and to crash the kidney type vortex.

Kuya et al. [12] studied the effect of swirled film cooling air on film cooling effectiveness by using a twisted tape in a circular cooling hole and found the improvement of film cooling effectiveness with swirling coolant flow. However, there was no description and experimental results that could be used to understand the mechanism of the film cooling with swirling flow.

In this study, a swirling motion of coolant flows was realized by two staggeredly-facing impinging jets with a slant angle, which give angular momentum to the coolant air inside the plenum chamber. To examine the effect of the swirl on the film cooling effectiveness, the film cooling effectiveness on the wall was measured by a pressure sensitive paint (PSP) technique for circular and shaped holes, and the spatial distribution of the film cooling effectiveness and flow field were measured by leaser induced fluorescence (LIF) and particle image velocimetry (PIV), respectively.

\section{Experimental apparatus and method}

\subsection{Wind tunnel and film cooling models}

The present experiment has been conducted using a scale-up model of film cooling holes installed on the bottom surface of a low-speed wind tunnel in order to allow detailed probing of flow features. The wind tunnel is an open-circuit and subsonic flow that can be produced through an inlet nozzle with a 9:1 contraction ratio. At the inlet of the nozzle section, there were honeycomb and a screen. 
Therefore, the airflow has low turbulence intensity and uniform velocity profile at the entrance of the test section. The test section is $300 \mathrm{~mm}$ wide, $300 \mathrm{~mm}$ height, and $1950 \mathrm{~mm}$ long. The air speed inside the test section can be varied from 0 to $40 \mathrm{~m} / \mathrm{s}$. For the free-stream velocity of $20 \mathrm{~m} / \mathrm{s}$, the flow at the test section shows excellent spatial uniformity, with free stream turbulence intensity less than $0.36 \%$. A film cooling hole is located $950 \mathrm{~mm}$ downstream from the exit of the contraction section. A schematic view of the wind tunnel is shown in Figure 1.

Figure 2 shows the geometry of film cooling holes, whose shapes are similar shapes to those used by Takeishi and Aoki [7]. They were made of low thermal conductivity material (Sanyo-chemical SanmodurMS) to reinforce the adiabatic condition. The guide channels to the exits of the film cooling holes were inclined at 30 degree towards the main flow direction. The shaped hole is composed of a round tube section with a uniformly and symmetrically expanded exit in the end, where a fan-shaped diffuser exists with 15-degree divergence angles on both lateral sides. The diameter of the guide channel, $d$, was $5 \mathrm{~mm}$. The coordinate axes and the origins are also shown in Figure 2.

Swirling coolant flow is made in a cavity that is located in a plenum before entering into a film cooling hole. A schematic view of the cooling structure is shown in Figure 3. The cross-section shape of the cavity is hexagonal, because it

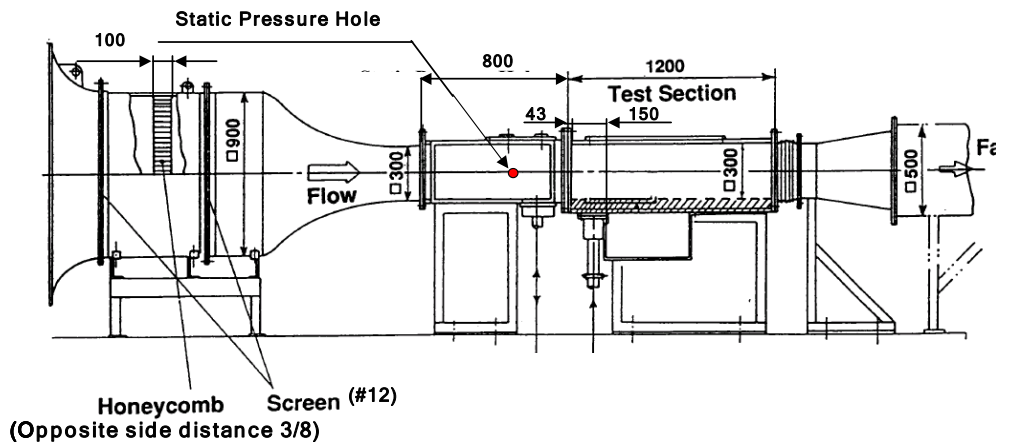

Figure 1: $\quad$ Schematic view of the wind tunnel.

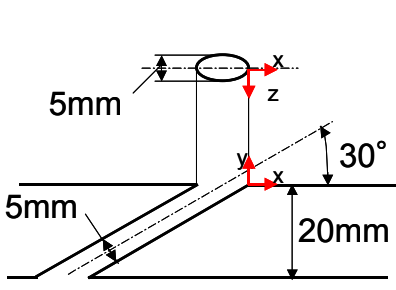

circular film cooling hole

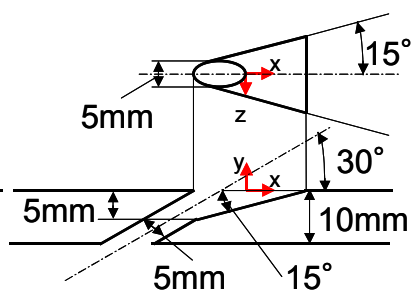

Shaped film cooling hole

Figure 2: Geometries of film cooling holes. 


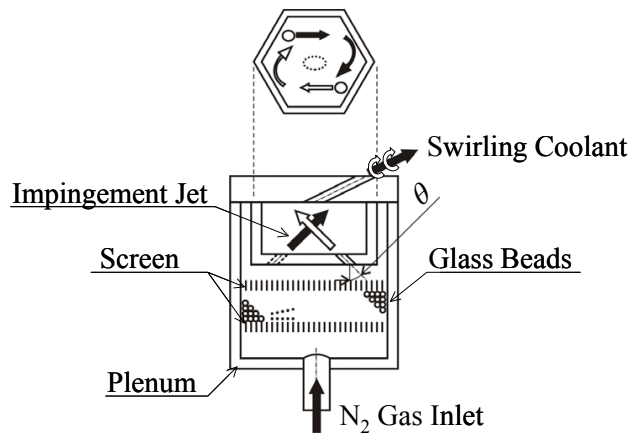

Figure 3: $\quad$ Structure of film cooling with swirling flow.

Table 1: $\quad$ Experimental conditions.

\begin{tabular}{|c|c|}
\hline Mainstream velocity [m/s] & 20 \\
\hline Turbulence intensity [\%] & 0.36 \\
\hline Boundary layer thickness [mm] & 25 \\
\hline Blowing ratio & $0.5-2.0$ \\
\hline Film cooling hole diameter [mm] & 5 \\
\hline Impingement jet angle [degree] & $0,10,20,30$ \\
\hline
\end{tabular}

can be set successively without void space in real applications. It has two impingement jet holes, and they are inclined at $\theta$ degree towards the vertical direction. Two impingement jets generate swirling flows inside the cavity, and this swirling flow enters into a film cooling hole, keeping the angular momentum. Swirl Number $S$ at the exit of the film cooling holes was measured by the PIV method. In the present study, two impingement jets angles were changed as $\theta=0,10,20,30$, and the corresponding Swirl Numbers were $S=0$, $0.0289,0.116$ and 0.168 , respectively. The experimental conditions are shown in Table 1.

\subsection{PSP methods}

In this study, PSP was used to measure film cooling effectiveness distribution on the wall. PSP is an optical pressure sensor. It uses a special pigment that changes its luminescence intensity by the reaction with oxygen molecules. The change of luminescence intensity is caused by optical quenching of the pigment by oxygen molecules. Therefore, we can measure a change of the concentration of oxygen by the luminescence intensity of the pigment. By using air (concentration of oxygen is $21 \%$ ) for mainstream and nitrogen (concentration of oxygen is $0 \%$ ) for the film cooling jet, the concentration distribution of oxygen, which is determined by the mixing of mainstream (air) and coolant (nitrogen), can be measured on the wall downstream of the film cooling hole. The film cooling effectiveness was defined by the following equation: 


$$
\eta=\frac{T_{\infty}-T_{f}}{T_{\infty}-T_{c}}
$$

where $T_{\infty}$ and $T_{c}$ are temperatures of the mainstream and coolant in the plenum respectively; $T_{f}$ is the wall temperature.

Then, using an analogy between heat/mass transfers, Eq. (1) is changed to Eq. (2).

$$
\eta=\frac{C_{\infty}-C_{f}}{C_{\infty}-C_{c}}
$$

The blowing ratio, which is defined as Eq. (3) was controlled by changing the film cooling flow rate.

$$
M=\frac{\rho_{c} u_{c}}{\rho_{\infty} u_{\infty}}
$$

The wall of wind tunnel is painted with PSP (ISSI PtTfPP FIB-UF405). Nitrogen gas for film cooling is supplied from nitrogen gas tank. LED light sources were used as an excitation light source. The images were taken with a CCD camera (Hamamatsu photonics C9440-05C 1344×1024 pixels, 12bit) located at the opposite side of PSP painted wall. Here, the light except phosphorescence (wave length $650 \mathrm{~nm}$ ) was cut by a band pass filter. The images were digitally stored on hard disks using the acquisition software HIPIC 8.30. The three images, a background image, an image when wind tunnel was pausing and an image when wind tunnel was running, were captured. 20 images were captured in each case, and they were averaged. PSP's luminescence intensity is affected by its temperature and hence the temperature of the wall during experiment was monitored by a K-type thermocouple. In the plenum, there were screen and glass beads and the airflow has a uniform velocity profile before entering into the film cooling hole.

\subsection{Laser induced fluorescence (LIF)}

LIF is a method for measuring mass concentration in gaseous flows. The advantages of LIF consist in being non-intrusive, instantaneous, and having high intensity of the fluorescence. In addition, LIF can realize high spatial resolution for the tracer concentration field. The flow is illuminated by a laser sheet of a wavelength that is tuned to excite a specific absorption transition of a molecular tracer, which is added for this purpose. A fraction of the molecules in the lower energy level absorbs the incident light and is excited to a higher energy state. When the excited state returns to the states with lower energy, fluorescence light is emitted with a different wavelength from that of the incident light. Thus, the fluorescence light from the tracer is extracted easily from the scattered light by using an interference filter.

If the thermal diffusivity of air and the mass diffusivity of a tracer are close, the thermal diffusion can be replaced by mass transfer based on heat/mass 
transfer analogy. Thus, local film cooling effectiveness $\eta$ can be replaced by equation (2).

Lozano et al. [13] showed that acetone is known as one of the tracers for concentration measurements in gaseous flows by LIF, and the ratio between acetone vapour's mass diffusion coefficient through air, $D$, and the thermal diffusivity of standard air, $\alpha$, is about one to two $\left(D=11.2 \mathrm{~mm}^{2} / \mathrm{s}, \alpha=22.3\right.$ $\mathrm{mm}^{2} / \mathrm{s}$ ). This is close enough to apply the heat/mass transfer analogy. In addition, acetone LIF is known to show good linearity with respect to the partial pressure of acetone in the atmospheric pressure gas.

The Nd:YAG laser at $266 \mathrm{~nm}$ was used as excitation laser light. The beam was expanded to a sheet of $25 \mathrm{~mm}$ width and $1 \mathrm{~mm}$ thickness with four cylindrical lenses, passing through the test section, as shown in Figure 4. The saturated acetone vapour in air was produced by bubbling the air in bottles containing acetone liquid. In order to obtain optimal acetone vapour concentration, dry air is mixed with air saturated with acetone vapour in a scheduled ratio [14-16].

\subsection{Particle image velocimetry (PIV)}

PIV was used to capture instantaneous velocity fields. The secondary flow blowing through film cooling holes contains fine particles of olive oil as a tracer, which are $1 \mu \mathrm{m}$ in diameter. The particles of olive oil were generated by bubbling the compressed air into a pool of olive oil through a Raskin nozzle in a pressure vessel. In the vessel, air bubbles burst at the olive-oil/air interface, and $1 \mu \mathrm{m}$ olive oil particles are released into the air. A dual-pulsed Nd:YAG laser, of wavelength $532 \mathrm{~nm}$, was employed to illuminate the tracers. Three cylindrical lenses were used to form a laser sheet. The laser sheet was guided by a mirror

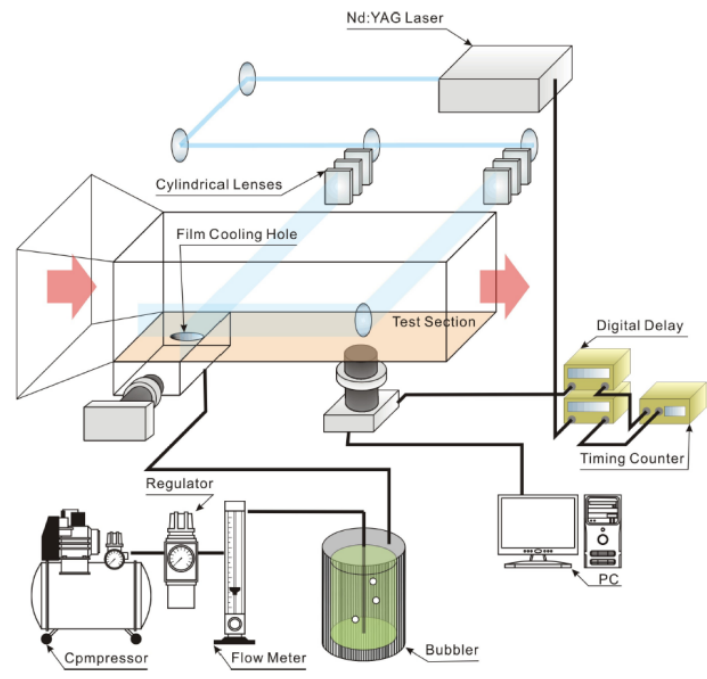

Figure 4: Schematic layout of acetone LIF method. 
located downstream to illuminate the test window. The particle pattern formed by the light reflected on the particle surfaces were taken with a CCD camera located at the side of the test section and the images were digitally stored on hard disks using the acquisition software HIPIC 8.0. To reduce the effect of reflection from the bottom surface, a background image for each pulse is subtracted from each frame to eliminate the effect of laser light reflection. The pairs of captured images were processed by a recursive local-correlation method to obtain velocity vector fields.

\section{Results and discussion}

\subsection{PSP measurements results}

Figures 5 and 6 show the film cooling effectiveness contours of a circular and a shaped holes at $M=1.0$ measured by PSP method with Swirl Number $S=0$ to 0.168. For non-swirling flow with a circular hole shown in figure 5 , the film
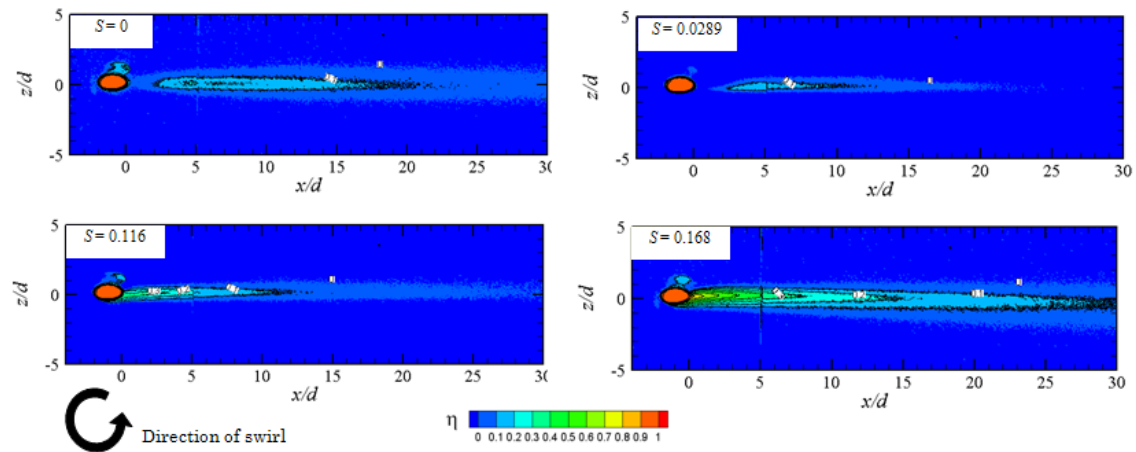

Figure 5: Film cooling effectiveness measured by PSP (circular hole, $\mathrm{M}=1.0)$.
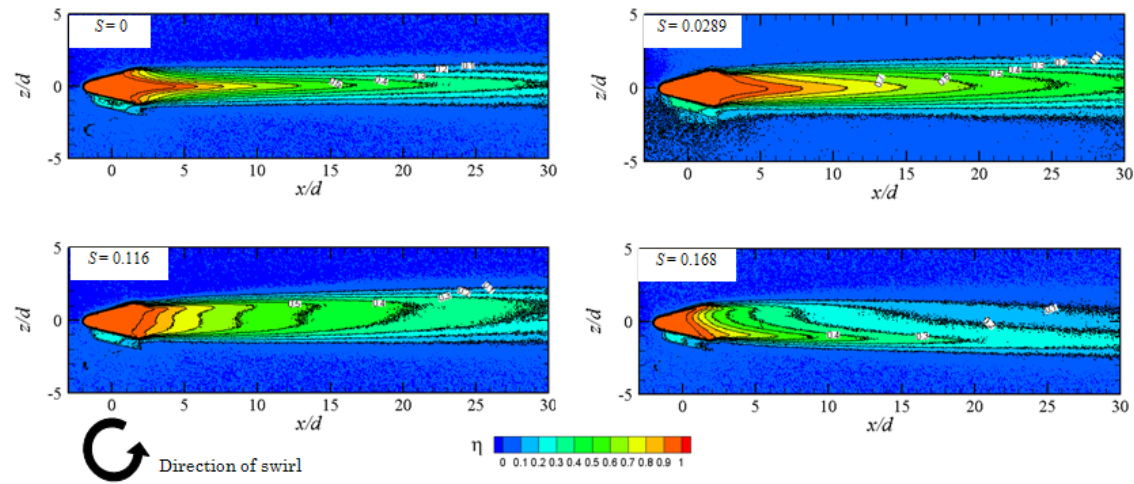

Figure 6: Film cooling effectiveness measured by PSP (shaped hole, $M=1.0$ ). 
cooling effectiveness just downstream the film cooling hole shows very low value. The reason of this low value is the penetration of film cooling jet into the main stream and the separation from the wall. At the downstream, the film turned to the wall directed by the mainstream and reattached on the surface near at $x / d=3$ and the film cooling effectiveness recovers to about 0.2. At the Swirl Number of $S=0.0289$, the area covered by film cooling air is decreased. However, further increase of Swirl Number suppressed the penetration of the film cooling jet and high film cooling effective area appeared just downstream of the film cooling hole exit. At $S=0.168$, highest film cooling effectiveness of about 0.6 is marked just downstream the film cooling hole exit. The result indicates that the penetration of the film cooling jet into the main stream was suppressed strongly by swirling motion of the film cooling air. It appeared that the centre line of the cooling jet flow was deflected to the negative $\mathrm{z}$ direction at the film cooling hole exit. Considering the relation between the swirling direction and the deflected direction of film jet, we found that the cooling jet moved to the same direction with the main stream. This bending force is thought to be Magnus effect.

Comparison of figures 5 and 6 shows that the film cooling jet blowing through a shaped cooling hole spreads wider both lateral direction and long distance.

Shaped film cooling attains higher film cooling effectiveness than circular hole by incorporation of exit shaping to the film holes, which results in lower momentum coolant injection with greater surface coverage. It appeared that the film cooling effectiveness reaches the maximum at $S=0.0289$ and decreased with a smaller or larger Swirl Number.

Figure 7 shows the spanwise averaged film cooling effectiveness for a circular hole and a shaped film cooling holes at $M=1.0$ with the Swirl Number $S$ $=0$ to 0.168 . Left figure in figure 7 shows that swirling coolant at $S=0.168$ improved the averaged film cooling effectiveness about $50 \%$ at $x / d=10$ and $100 \%$ or more at far downstream of $x / d=20$.
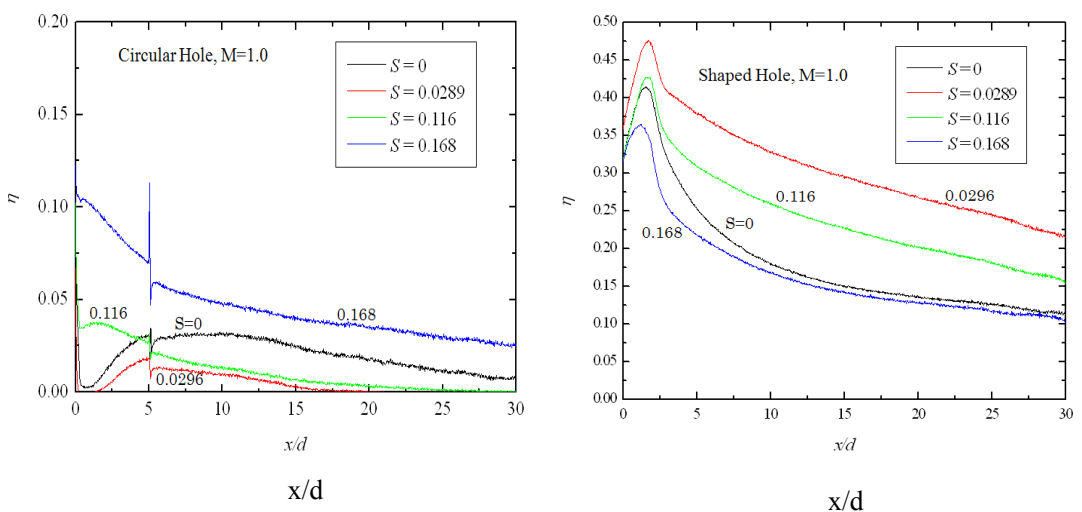

Figure 7: $\quad$ Spanwise averaged film cooling effectiveness. 
It appeared from left figure in figure 7 that swirling coolant flow is very effective to improve the film cooling effectiveness for circular hole by suppressing both the penetration of cooling jet into mainstream and the generation of anti-kidney vortex structure. Right figure in figure 7 shows that swirling coolant also improved the averaged film cooling effectiveness of shaped film cooling hole about $100 \%$ at far down of $x / d=10$.

There should be an optimum combination of shaped hole geometry and Swirl Number. In this case of the geometry shown in figure 2, the averaged film cooling effectiveness achieves the maximum at $S=0.0289$.

\subsection{LIF measurement results}

Figures 8 to 10 show the time-averaged film cooling effectiveness distribution at $z / d=0$ and the cross sections at selected locations obtained by acetone LIF near the exit of a circular hole and a shaped hole, for blowing ratios of $M=1.0$. For the circular hole, the penetration of the film cooling jet into mainstream was suppressed by swirling coolant flow and attained higher film cooling effectiveness compared to the non-swirling coolant flow case.

Figure 9 clearly shows the generation of anti-kidney vortex structure at $S=0$. Comparison of the measured LIF results of $S=0$ and $S=0.168$ shows that the swirling film cooling flow interacts with the mainstream and suppresses the formation of the anti-kidney vortex structure and this results in the coolant adhesion on the wall.

Figure 10 shows the measured cross section film cooling effectiveness at $S=$ 0 and 0.0289 . As shown in right figure of figure 7, the film cooling effectiveness attains maximum value at $S=0.0289$ with the shaped geometry shown in figure 2. It is clear from figure 10 that the swirling film cooling flow suppressed the penetration or diffusion of film cooling air to y-direction and spreads the film cooling air in spanwise direction and, as a result, covers wide area on the wall, and attains highest film cooling effectiveness.
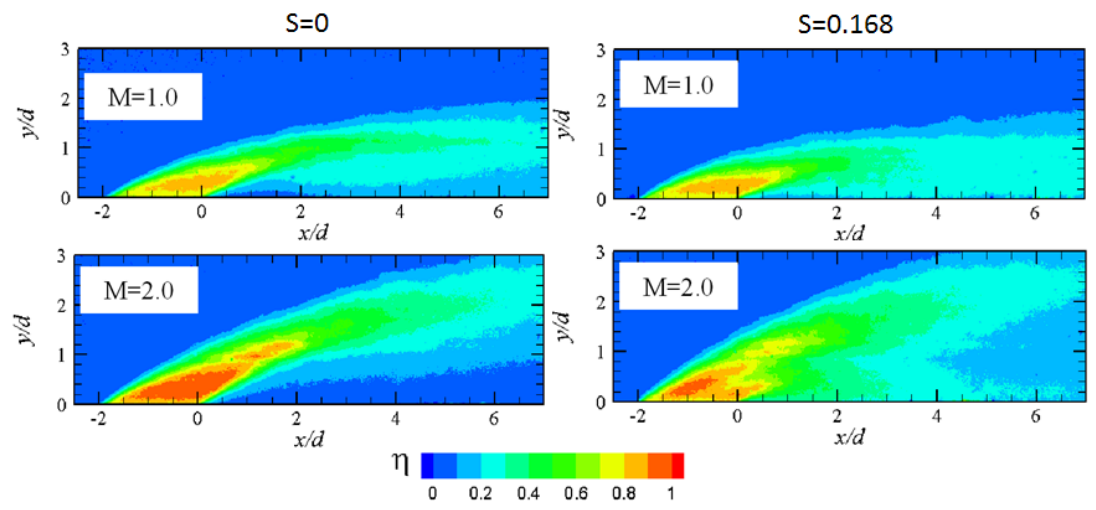

Figure 8: Film cooling effectiveness at $z / d=0$ section (circular hole). 

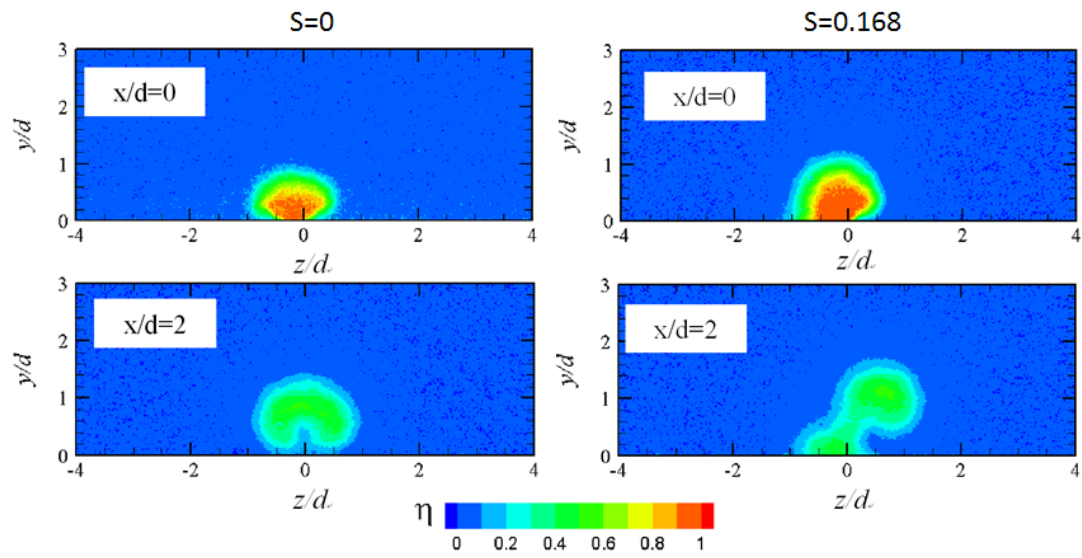

Figure 9: Cross section film cooling effectiveness (circular hole, $M=1.0$ ).
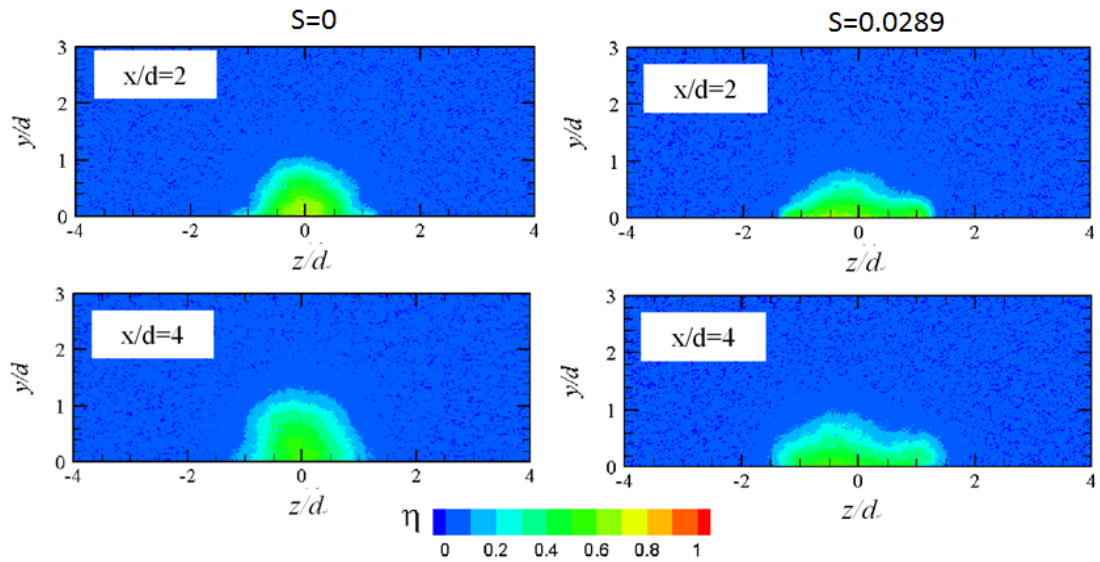

Figure 10: Cross section film cooling effectiveness (shaped hole, $M=1.0$ ).

\subsection{PIV results}

Figures 11 and 12 show the time-averaged velocity vectors and vorticity distribution measured for circular film cooling hole at $M=1.0$ by PIV method. The generation of anti-kidney vortex structure is affected by the swirling motion of film cooling flow at $S=0.168$, and film cooling air adheres on the wall. As it is clear from figure 12, a quite low velocity region and a shear layer was detected behind the circular film cooling hole exit, but adding swirling motion to film cooling the film cooling hole exit at $S=0$. However, penetration of the film cooling jet into mainstream was suppressed and coolant adhered on the wall at $S$ $=0.168$. 


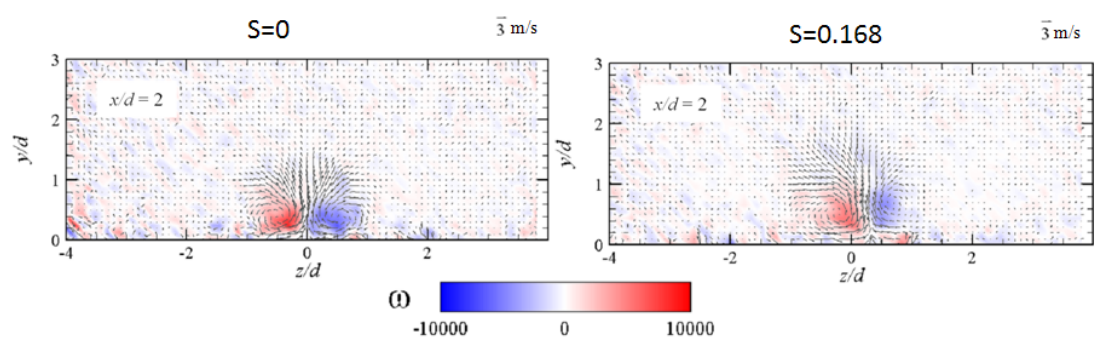

Figure 11: Time-mean velocity field of the circular hole $(\mathrm{M}=1.0)$.

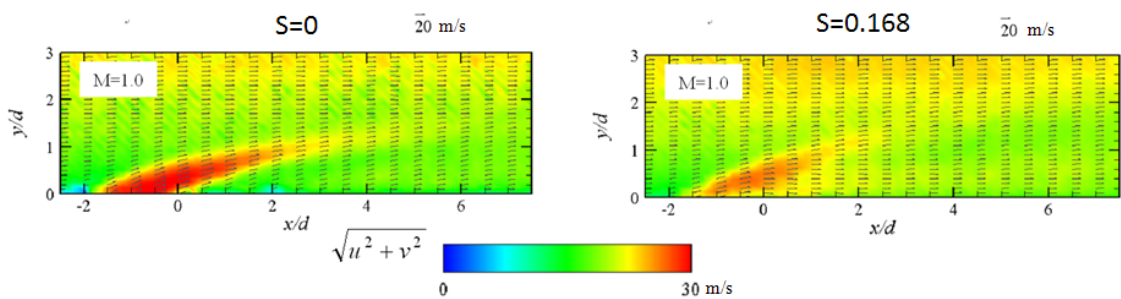

$\overrightarrow{2} 0 \mathrm{~m} / \mathrm{s} \quad \overrightarrow{2} 0 \mathrm{~m} / \mathrm{s}$

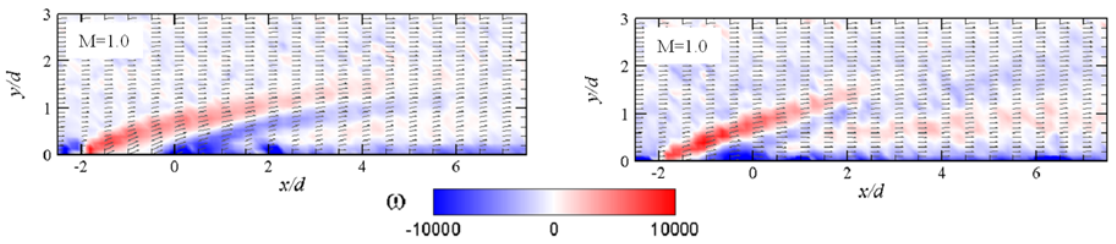

Figure 12: Time-mean velocity vector at $z / d=0$ and time-mean velocity field (circular hole).

\section{Conclusions}

The new invention to improve film cooling effectiveness by using swirling film cooling flow applied to circular and shaped film cooling holes. The following conclusions were obtained through these experimental works.

1. Film cooling with swirling flow improved drastically the film cooling effectiveness blowing through a circular hole. This improvement was attained by the interaction between swirling film jet and mainstream, and swirling motion destructed the anti-kidney vortex structure and made the film cooling air adhere on the wall.

2. Application of swirling flow to shaped film cooling was also drastically effective to improve film cooling effectiveness. But there is the optimum combination between a shaped hole geometry and Swirl Number.

3. Acetone LIF is a very useful method to investigate such complicated mixing phenomena quantitatively and the results are useful to evaluate the accuracy of numerical works. 


\section{References}

[1] Goldstein, R. J., Film Cooling, Advances in Heat Transfer, Vol. 7, pp. 321379. 1971.

[2] Bogard, D. G. and Thole, K. A., Gas Turbine Film Cooling, J. of Propulsion and Power, 22, pp. 249-270, 2006.

[3] Eriksen, V, L. and Goldstein, R. J., Heat Transfer and Film Cooling Following Injection Through Inclined Circular Tubes, J. of Heat Transfer, pp. 239-245, 1974.

[4] Bernsdorf, S., Rose, M. G. and Abhari, R. S., Modeling of Film Cooling Part I: Experimental Study of Flow Structure, ASME Paper No. GT200568783, 2005.

[5] Goldstein, R. J. and Eckert, E. R. G., Effect of hole geometry and density on three-dimensional film cooling, Int. J. Heat and Mass Transfer, 17, pp. 595607, 1994.

[6] Bell, C. M., Hamakawa, H. and Ligrani, P. M., Film Cooling From Shaped Holes, J. of Turbomachinery, Vol. 122, pp. 224-232, 2000.

[7] Takeishi, K., and Aoki, S., Contribution of Heat Transfer to Turbine Blades and Vanes for High Temperature Industrial Gas Turbines: Part 1, Film Cooling, Ann. N. Y. Acad. Sci., Vol. 934, pp. 305-312. 2001.

[8] Yu, Y, Yen, C. -H. Shin, T. I. -P. and Chyu, M. K. Film Cooling Effectiveness and Heat Transfer Coefficient Distribution Around Diffusion Shaped Holes, J. of Heat Transfer, Vol. 124, pp. 820-827. 2000.

[9] Bunker, R. S., A Review of Shaped Hole Turbine Film-Cooling Technology, J. of Heat Transfer, Vol. 127, pp. 441-453. 2005.

[10] Kusterer, K., et al., A parametric study on the influence of the lateral ejection angle of double-jet holes on the film cooling effectiveness for high blowing ratios, ASME Paper GT2009-59321, 2009.

[11] Heidmann, J. D. and Ekkad, S., A novel anti-votex turbine film- cooling hole concept, ASME paper, GT2007-27528, 2007.

[12] Kuya, Y., Nuntadusit, C., Ishida, H., Momose, K. and Kimoto, H., An Application of Swirling Jet to Film Cooling, Proc., JSME Thermal Engineering Conference, Sendai, No.04-28, (in Japanese), 2004.

[13] Lazano, A., Yip, B. and Hanson, R. K., Acetone: a tracer for concentration measurements in gaseous flows by planer laser-induced fluorescence, Experiments in Fluids, Vol. 13, 369-376, 1992.

[14] Kumagai, K., Takeishi, K., Komiyama, M. and Tokunaga, D., Numerical and experimental research on a mixing process of film cooling air with mainstream, Proc., Inter. Heat Transfer Conference, No. FCV-14, 2006.

[15] Kajiuchi, T., Takeishi, K., Oda, Y., and Kumagai, T., Numerical and Experimental Research on the Film Cooling Flow Fields from Circular and Shaped Holes, Proc., Inter. Gas Turbine Congress, Paper No. IGTC7 Tokyo TS-109, 2007.

[16] Takeishi, K., Kitamura, T., Komiyama, M., Oda, Y. and Mori, S., Study on the thermal and flow fields of shaped film cooling holes, Int. Symp. on Heat Transfer in Gas Turbine Systems, Antalya, Turkey, 2009. 